Analele Universităţii de Vest, Timişoara

Seria Matematică - Informatică

LIV , 1, (2016), 73-86

\title{
Positive Solutions of a Nonlinear Fourth-order Integral Boundary Value Problem
}

Slimane Benaicha and Faouzi Haddouchi

\begin{abstract}
In this paper, the existence of positive solutions for a nonlinear fourth-order two-point boundary value problem with integral condition is investigated. By using Krasnoselskii's fixed point theorem on cones, sufficient conditions for the existence of at least one positive solutions are obtained.
\end{abstract}

AMS Subject Classification (2000). 34B15; 34B18

Keywords. Positive solutions, Krasnoselskii's fixed point theorem, fourth-order integral boundary value problems, existence, cone.

\section{Introduction}

In this work, we study the existence of positive solutions of a nonlinear two-point boundary value problem (BVP) for the following fourth-order differential equation:

$$
\begin{gathered}
u^{\prime \prime \prime \prime}(t)+f(u(t))=0, t \in(0,1), \\
u^{\prime}(0)=u^{\prime}(1)=u^{\prime \prime}(0)=0, u(0)=\int_{0}^{1} a(s) u(s) d s,
\end{gathered}
$$

where 
(H1) $f \in C([0, \infty),[0, \infty))$;

(H2) $a \in C([0,1],[0, \infty))$ and $0<\int_{0}^{1} a(s) d s<1$.

Fourth-order ordinary differential equations are models for bending or deformation of elastic beams, and therefore have important applications in engineering and physical sciences. Recently, the two-point and multi-point boundary value problems for fourth-order nonlinear differential equations have received much attention from many authors. Many authors have studied the beam equation under various boundary conditions and by different approaches. In 2009, Graef et al.[5] considered the fourth order three-point boundary value problem

$$
u^{\prime \prime \prime \prime}(t)=g(t) f(u(t)), t \in(0,1),
$$

together with the boundary conditions

$$
u(0)=u^{\prime}(0)=u^{\prime \prime}(\beta)=u^{\prime \prime}(1)=0 .
$$

In 2006, Anderson and Avery [2], studied the following fourth order right focal four-point boundary value problem

$$
\begin{gathered}
u^{\prime \prime \prime \prime}(t)+f(u(t))=0,0<t<1, \\
u(0)=u^{\prime}(q)=u^{\prime \prime}(r)=u^{\prime \prime \prime}(1)=0 .
\end{gathered}
$$

In 2011, Xiading Han et al.[6], considered the nonlocal fourth-order boundary value problem with variable parameter

$$
\begin{gathered}
u^{\prime \prime \prime \prime}(t)+B(t) u^{\prime \prime}(t)=\lambda f\left(t, u(t), u^{\prime \prime}(t)\right), 0<t<1, \\
u(0)=u(1)=\int_{0}^{1} p(s) u(s) d s, u^{\prime \prime}(0)=u^{\prime \prime}(1)=\int_{0}^{1} q(s) u^{\prime \prime}(s) d s .
\end{gathered}
$$

In 2013, Yan Sun and Cun Zhu [14], considered the singular fourth-order three-point boundary value problem

$$
\begin{gathered}
u^{\prime \prime \prime \prime}(t)+f(t, u(t))=0,0<t<1, \\
u(0)=u^{\prime}(0)=u^{\prime \prime}(0)=0, u^{\prime \prime}(1)-\alpha u^{\prime \prime}(\eta)=\lambda .
\end{gathered}
$$

In 2014, Xiaorui Liu and Dexiang Ma [10], considered the third-order twopoint boundary value problem

$$
u^{\prime \prime \prime}(t)+f(u(t))=0,0<t<1,
$$




$$
u^{\prime}(0)=u^{\prime}(1)=0, u(0)=\int_{0}^{1} k(s) u(s) d s,
$$

and in 2015, Wenguo shen [13], studied the fourth-order second-point nonhomogeneous singular boundary value problem

$$
\begin{gathered}
u^{\prime \prime \prime \prime}(t)+a(t) f(u(t))=0,0<t<1, \\
u(0)=\alpha, u(1)=\beta, u^{\prime}(0)=\lambda, u^{\prime}(1)=-\mu .
\end{gathered}
$$

For some other results on boundary value problem, we refer the reader to the papers $[1,3,4,7,8,11,12,15-19]$. To the authors' knowledge, there are few papers that have considered the existence of solutions for fourth-order two-point boundary value problem with integral condition. Motivated by the works mentioned above, the aim of this paper is to establish some sufficient conditions for the existence of at least one positive solutions of the BVP (1.1) and (1.2).

We shall first construct the Green's function for the associated linear boundary value problem and then determine the properties of the Green's function for associated linear boundary value problem. Finally, existence results for at least one positive solution for the above problem are established when $f$ is superlinear or sublinear. As applications, some interesting examples are presented to illustrate the main results.

\section{Preliminaries}

We shall consider the Banach space $C([0,1])$ equipped with the sup norm

$$
\|u\|=\sup _{t \in[0,1]}|u(t)|
$$

Definition 2.1. Let $E$ be a real Banach space. A nonempty, closed, convex set $K \subset E$ is a cone if it satisfies the following two conditions:

(i) $x \in K, \lambda \geq 0$ imply $\lambda x \in K$;

(ii) $x \in K,-x \in K$ imply $x=0$.

Definition 2.2. An operator $T: E \rightarrow E$ is completely continuous if it is continuous and maps bounded sets into relatively compact sets.

To prove some of our results, we will use the following fixed point theorem, which is due to Krasnoselskii's [9]. 
Theorem 2.1. [9]. Let $E$ be a Banach space, and let $K \subset E$, be a cone. Assume that $\Omega_{1}$ and $\Omega_{2}$ are open subsets of $E$ with $0 \in \Omega_{1}, \Omega_{1} \subset \Omega_{2}$ and let

$$
A: K \cap\left(\overline{\Omega_{2}} \backslash \Omega_{1}\right) \rightarrow K
$$

be a completely continuous operator such that

(i) $\|A u\| \leq\|u\|, u \in K \cap \partial \Omega_{1}$, and $\|A u\| \geq\|u\|, u \in K \cap \partial \Omega_{2}$; or

(ii) $\|A u\| \geq\|u\|, u \in K \cap \partial \Omega_{1}$, and $\|A u\| \leq\|u\|, u \in K \cap \partial \Omega_{2}$.

Then $A$ has a fixed point in $K \cap\left(\overline{\Omega_{2}} \backslash \Omega_{1}\right)$.

Consider the two-point boundary value problem

$$
\begin{gathered}
u^{\prime \prime \prime \prime}(t)+y(t)=0, t \in(0,1), \\
u^{\prime}(0)=u^{\prime}(1)=u^{\prime \prime}(0)=0, u(0)=\int_{0}^{1} a(s) u(s) d s .
\end{gathered}
$$

Lemma 2.2. The problem (2.1)-(2.2) has a unique solution

$$
u(t)=\int_{0}^{1}\left(G(t, s)+\frac{1}{1-\alpha} \int_{0}^{1} a(\tau) G(\tau, s) d \tau\right) y(s) d s
$$

where $G(t, s):[0,1] \times[0,1] \rightarrow \mathbb{R}$ is the Green's function defined by

$$
G(t, s)=\frac{1}{6} \begin{cases}t^{3}(1-s)^{2}-(t-s)^{3}, & 0 \leq s \leq t \leq 1 \\ t^{3}(1-s)^{2}, & 0 \leq t \leq s \leq 1\end{cases}
$$

and

$$
\alpha=\int_{0}^{1} a(t) d t
$$

Proof. Integrating (2.1) over the interval $[0, t]$ for $t \in[0,1]$, we obtain

$$
\begin{gathered}
u^{\prime \prime \prime}(t)=-\int_{0}^{t} y(s) d s+C_{1}, \\
u^{\prime \prime}(t)=-\int_{0}^{t}(t-s) y(s) d s+C_{1} t+C_{2}, \\
u^{\prime}(t)=-\frac{1}{2} \int_{0}^{t}(t-s)^{2} y(s) d s+\frac{1}{2} C_{1} t^{2}+C_{2} t+C_{3}, \\
u(t)=-\frac{1}{6} \int_{0}^{t}(t-s)^{3} y(s) d s+\frac{1}{6} C_{1} t^{3}+\frac{1}{2} C_{2} t^{2}+C_{3} t+C_{4} .
\end{gathered}
$$


Vol. LIV (2016) Positive solutions of a fourth-order ...

From the boundary conditions (2.2) we get

$$
C_{2}=C_{3}=0, \quad C_{1}=\int_{0}^{1}(1-s)^{2} y(s) d s,
$$

and

$$
\begin{aligned}
C_{4}= & u(0) \\
= & \int_{0}^{1} a(\tau)\left(-\frac{1}{6} \int_{0}^{\tau}(\tau-s)^{3} y(s) d s+\frac{1}{6} \tau^{3} \int_{0}^{1}(1-s)^{2} y(s) d s+C_{4}\right) d \tau \\
= & C_{4} \int_{0}^{1} a(\tau) d \tau-\frac{1}{6} \int_{0}^{1} a(\tau)\left(\int_{0}^{\tau}(\tau-s)^{3} y(s) d s\right) d \tau \\
& +\frac{1}{6} \int_{0}^{1} a(\tau) \tau^{3}\left(\int_{0}^{1}(1-s)^{2} y(s) d s\right) d \tau,
\end{aligned}
$$

which implies

$$
\begin{aligned}
C_{4}= & \frac{1}{6(1-\alpha)}\left(\int_{0}^{1} a(\tau) \tau^{3}\left(\int_{0}^{1}(1-s)^{2} y(s) d s\right) d \tau\right. \\
& \left.-\int_{0}^{1} a(\tau)\left(\int_{0}^{\tau}(\tau-s)^{3} y(s) d s\right) d \tau\right) .
\end{aligned}
$$

Replacing these expressions in (2.4), we get

$$
\begin{aligned}
u(t)= & -\frac{1}{6} \int_{0}^{t}(t-s)^{3} y(s) d s+\frac{1}{6} t^{3} \int_{0}^{1}(1-s)^{2} y(s) d s \\
& +\frac{1}{6(1-\alpha)}\left[\int_{0}^{1} a(\tau) \tau^{3}\left(\int_{0}^{1}(1-s)^{2} y(s) d s\right) d \tau\right. \\
& \left.-\int_{0}^{1} a(\tau)\left(\int_{0}^{\tau}(\tau-s)^{3} y(s) d s\right) d \tau\right] \\
= & \frac{1}{6} \int_{0}^{t}\left[t^{3}(1-s)^{2}-(t-s)^{3}\right] y(s) d s+\frac{1}{6} \int_{t}^{1} t^{3}(1-s)^{2} y(s) d s \\
& +\frac{1}{6(1-\alpha)}\left[\int _ { 0 } ^ { 1 } a ( \tau ) \left(\int_{0}^{\tau}\left[\tau^{3}(1-s)^{2}-(\tau-s)^{3}\right] y(s) d s\right.\right. \\
& \left.\left.+\int_{\tau}^{1} \tau^{3}(1-s)^{2} y(s) d s\right) d \tau\right]
\end{aligned}
$$




$$
\begin{aligned}
= & \frac{1}{6} \int_{0}^{t}\left[t^{3}(1-s)^{2}-(t-s)^{3}\right] y(s) d s+\frac{1}{6} \int_{t}^{1} t^{3}(1-s)^{2} y(s) d s \\
& +\frac{1}{1-\alpha} \int_{0}^{1}\left(\frac{1}{6} \int_{0}^{\tau} a(\tau)\left[\tau^{3}(1-s)^{2}-(\tau-s)^{3}\right] y(s) d s\right. \\
& \left.+\frac{1}{6} \int_{\tau}^{1} a(\tau) \tau^{3}(1-s)^{2} y(s) d s\right) d \tau \\
= & \int_{0}^{1} G(t, s) y(s) d s+\frac{1}{1-\alpha} \int_{0}^{1}\left(\int_{0}^{1} a(\tau) G(\tau, s) y(s) d s\right) d \tau \\
= & \int_{0}^{1}\left(G(t, s)+\frac{1}{1-\alpha} \int_{0}^{1} a(\tau) G(\tau, s) d \tau\right) y(s) d s .
\end{aligned}
$$

Lemma 2.3. Let $\theta \in] 0, \frac{1}{2}[$ be fixed. Then

(i) $G(t, s) \geq 0$, for all $t, s \in[0,1]$;

(ii) $\frac{1}{6} \theta^{3} s(1-s)^{2} \leq G(t, s) \leq \frac{1}{6} s(1-s)^{2}$, for all $(t, s) \in[\theta, 1-\theta] \times[0,1]$.

Proof. (i) We will show that $G(t, s) \geq 0$, for all $(t, s) \in[0,1] \times[0,1]$. Since it is obvious for $t \leq s$, we only need to prove the case $s \leq t$. Now we suppose that $s \leq t$. Then

$$
\begin{aligned}
G(t, s) & =\frac{1}{6}\left[t^{3}(1-s)^{2}-(t-s)^{3}\right]=\frac{1}{6}\left[t(t-t s)^{2}-(t-s)^{3}\right] \\
& \geq \frac{1}{6}\left[t(t-s)^{2}-(t-s)^{3}\right] \\
& \geq \frac{1}{6}(t-s)^{2}[t-(t-s)] \\
& =\frac{1}{6} s(t-s)^{2} \geq 0
\end{aligned}
$$

(ii) If $s \leq t$, from $(2.3)$ we have

$$
\begin{aligned}
G(t, s) & =\frac{1}{6}\left[t^{3}(1-s)^{2}-(t-s)^{3}\right] \geq \frac{1}{6}\left[t^{3}(1-s)^{3}-(t-s)^{3}\right] \\
& =\frac{1}{6}\left[(t-t s)^{3}-(t-s)^{3}\right] \\
& =\frac{1}{6} s(1-t)\left[t^{2}(1-s)^{2}+t(1-s)(t-s)+(t-s)^{2}\right] \\
& \geq \frac{1}{6} t^{2}(1-t) s(1-s)^{2} .
\end{aligned}
$$


On the other hand

$$
\begin{aligned}
G(t, s)-\frac{1}{6} s(1-s)^{2} & =\frac{1}{6} t^{3}(1-s)^{2}-\frac{1}{6}(t-s)^{3}-\frac{1}{6} s(1-s)^{2} \\
& =\frac{1}{6} s\left(-2 t^{3}+t^{3} s+3 t^{2}-3 t s-1+2 s\right) \\
& =\frac{1}{6} s(t-1)^{2}[(s-2) t+2 s-1] \\
& \leq \frac{1}{6} s(t-1)^{2}[(s-2) t+2 t-1] \\
& =\frac{1}{6} s(t-1)^{2}(s t-1) \leq 0
\end{aligned}
$$

If $t \leq s$, from (2.3), we have

$$
G(t, s)=\frac{1}{6} t^{3}(1-s)^{2} \geq \frac{1}{6} t^{3} s(1-s)^{2},
$$

and,

$$
G(t, s)=\frac{1}{6} t^{3}(1-s)^{2} \leq \frac{1}{6} s^{3}(1-s)^{2} \leq \frac{1}{6} s(1-s)^{2} .
$$

Let

$$
\rho(t)=\frac{1}{6} \min \left\{t^{3}, t^{2}(1-t)\right\}=\frac{1}{6} \begin{cases}t^{3}, & t \leq \frac{1}{2} \\ t^{2}(1-t), & t \geq \frac{1}{2}\end{cases}
$$

From (2.6), (2.7), (2.8) and (2.9) we have

$$
\rho(t) s(1-s)^{2} \leq G(t, s) \leq \frac{1}{6} s(1-s)^{2}, \text { for all }(t, s) \in[0,1] \times[0,1] .
$$

For $\theta \in] 0, \frac{1}{2}[$, we have

$$
\frac{\theta^{3}}{6} s(1-s)^{2} \leq G(t, s) \leq \frac{1}{6} s(1-s)^{2}, \text { for all }(t, s) \in[\theta, 1-\theta] \times[0,1] .
$$

Lemma 2.4. Let $y(t) \in C([0,1],[0, \infty))$ and $\theta \in] 0, \frac{1}{2}[$. The unique solution of (2.1)-(2.2) is nonnegative and satisfies

$$
\min _{t \in[\theta, 1-\theta]} u(t) \geq \theta^{3}(1-\alpha+\beta)\|u\|
$$

where $\beta=\int_{\theta}^{1-\theta} a(t) d t, \quad \alpha=\int_{0}^{1} a(t) d t$. 
Proof. From Lemma 2.2 and Lemma 2.3, $u(t)$ is nonnegative. For $t \in[0,1]$, from Lemma 2.2 and Lemma 2.3, we have that

$$
\begin{aligned}
u(t) & =\int_{0}^{1}\left(G(t, s)+\frac{1}{1-\alpha} \int_{0}^{1} a(\tau) G(\tau, s) d \tau\right) y(s) d s \\
& \leq \frac{1}{6} \int_{0}^{1} s(1-s)^{2}\left(1+\frac{\alpha}{1-\alpha}\right) y(s) d s \\
& =\frac{1}{6(1-\alpha)} \int_{0}^{1} s(1-s)^{2} y(s) d s .
\end{aligned}
$$

Then

$$
\|u\| \leq \frac{1}{6(1-\alpha)} \int_{0}^{1} s(1-s)^{2} y(s) d s,
$$

and for $t \in[\theta, 1-\theta]$, we have

$$
\begin{aligned}
u(t) & =\int_{0}^{1}\left(G(t, s)+\frac{1}{1-\alpha} \int_{0}^{1} a(\tau) G(\tau, s) d \tau\right) y(s) d s \\
& \geq \frac{\theta^{3}}{6} \int_{0}^{1}\left[s(1-s)^{2}+\frac{1}{1-\alpha} \int_{\theta}^{1-\theta} s(1-s)^{2} a(\tau) d \tau\right] y(s) d s \\
& =\frac{\theta^{3}}{6} \int_{0}^{1} s(1-s)^{2}\left(1+\frac{\beta}{1-\alpha}\right) y(s) d s \\
& =\frac{\theta^{3}}{6} \cdot \frac{1-\alpha+\beta}{1-\alpha} \int_{0}^{1} s(1-s)^{2} y(s) d s .
\end{aligned}
$$

From (2.10), (2.11), we obtain

$$
\min _{t \in[\theta, 1-\theta]} u(t) \geq \theta^{3}(1-\alpha+\beta)\|u\| .
$$

Let $\theta \in] 0, \frac{1}{2}[$. We define the cone

$$
K=\left\{u \in C([0,1], \mathbb{R}), u \geq 0: \min _{t \in[\theta, 1-\theta]} u(t) \geq \theta^{3}(1-\alpha+\beta)\|u\|\right\},
$$

and the operator $A: K \rightarrow C[0,1]$ by

$$
A u(t)=\int_{0}^{1}\left(G(t, s)+\frac{1}{1-\alpha} \int_{0}^{1} a(\tau) G(\tau, s) d \tau\right) f(u(s)) d s
$$


Remark 2.1. By Lemma 2.2, problem (1.1), (1.2) has a positive solution $u(t)$ if and only if $u$ is a fixed point of $A$.

Lemma 2.5. The operator A defined in (2.12) is completely continuous and satisfies $A K \subset K$.

Proof. From Lemma 2.4, we obtain $A K \subset K . A$ is completely continuous by an application of Arzela-Ascoli theorem.

In what follows, we will use the following notations

$$
f_{0}=\lim _{u \rightarrow 0+} \frac{f(u)}{u}, \quad f_{\infty}=\lim _{u \rightarrow \infty} \frac{f(u)}{u} .
$$

We note that the case $f_{0}=0$ and $f_{\infty}=\infty$ corresponds to the superlinear case and $f_{0}=\infty$ and $f_{\infty}=0$ corresponds to the sublinear case.

\section{$3 \quad$ Existence of positive solutions}

In this section, we will state and prove our main results.

Theorem 3.1. Assume that $f_{0}=0$ and $f_{\infty}=\infty$. Then $B V P(1.1)$ and (1.2) has at least one positive solution.

Proof. Since $f_{0}=0$, there exists $\rho_{1}>0$ such that $f(u) \leq \epsilon u$, for $0<u \leq \rho_{1}$, where $\epsilon>0$ satisfies

$$
\frac{\epsilon}{6(1-\alpha)} \leq 1
$$

Thus, if we let

$$
\Omega_{1}=\left\{u \in C[0,1]:\|u\|<\rho_{1}\right\},
$$

then, for $u \in K \cap \partial \Omega_{1}$, we have

$$
\begin{aligned}
A u(t) & =\int_{0}^{1}\left(G(t, s)+\frac{1}{1-\alpha} \int_{0}^{1} a(\tau) G(\tau, s) d \tau\right) f(u(s)) d s \\
& \leq \frac{1}{6} \int_{0}^{1}\left(s(1-s)^{2}+\frac{1}{1-\alpha} \int_{0}^{1} s(1-s)^{2} a(\tau) d \tau\right) \epsilon u(s) d s \\
& \leq \frac{1}{6} \cdot \frac{\epsilon}{1-\alpha}\|u\| \int_{0}^{1} s(1-s)^{2} d s \\
& \leq \frac{1}{6} \cdot \frac{\epsilon}{1-\alpha}\|u\| \\
& \leq\|u\| .
\end{aligned}
$$


Therefore

$$
\|A u\| \leq\|u\|, \quad u \in K \cap \partial \Omega_{1} .
$$

Further, since $f_{\infty}=\infty$, there exists $\widehat{\rho}_{2}>0$ such that $f(u) \geq \delta u$, for $u>\widehat{\rho}_{2}$, where $\delta>0$ is chosen so that

$$
\delta \frac{\theta^{6}}{36} \cdot \frac{(1-\alpha+\beta)^{2}}{1-\alpha}(1-2 \theta)\left(\frac{1}{2}+\theta-\theta^{2}\right) \geq 1 .
$$

Let $\rho_{2}=\max \left\{2 \rho_{1}, \frac{\widehat{\rho}_{2}}{\theta^{3}(1-\alpha+\beta)}\right\}$ and $\Omega_{2}=\left\{u \in C[0,1]:\|u\|<\rho_{2}\right\}$. Then $u \in K \cap \partial \Omega_{2}$ implies that

$$
\min _{t \in[\theta, 1-\theta]} u(t) \geq \theta^{3}(1-\alpha+\beta)\|u\|=\theta^{3}(1-\alpha+\beta) \rho_{2} \geq \widehat{\rho}_{2},
$$

so, by (2.12) and for $t \in[\theta, 1-\theta]$, we obtain

$$
\begin{aligned}
A u(t) & =\int_{0}^{1}\left(G(t, s)+\frac{1}{1-\alpha} \int_{0}^{1} a(\tau) G(\tau, s) d \tau\right) f(u(s)) d s \\
& \geq \int_{\theta}^{1-\theta}\left[\frac{\theta^{3}}{6} s(1-s)^{2}+\frac{1}{1-\alpha} \int_{\theta}^{1-\theta} \frac{\theta^{3}}{6} s(1-s)^{2} a(\tau) d \tau\right] \delta u(s) d s \\
& =\frac{\theta^{3}}{6} \delta \int_{\theta}^{1-\theta} s(1-s)^{2}\left(1+\frac{\beta}{1-\alpha}\right) u(s) d s \\
& =\frac{\theta^{3} \delta}{6} \cdot \frac{(1-\alpha+\beta)}{1-\alpha} \int_{\theta}^{1-\theta} s(1-s)^{2} u(s) d s \\
& \geq \frac{\theta^{3} \delta}{6} \cdot \frac{(1-\alpha+\beta)}{1-\alpha} \min _{t \in[\theta, 1-\theta]} u(t) \int_{\theta}^{1-\theta} s(1-s)^{2} d s \\
& \geq \delta \frac{\theta^{6}}{36} \cdot \frac{(1-\alpha+\beta)^{2}}{1-\alpha}(1-2 \theta)\left(\frac{1}{2}+\theta-\theta^{2}\right)\|u\| \\
& \geq\|u\| .
\end{aligned}
$$

Hence, $\|A u\| \geq\|u\|, u \in K \cap \partial \Omega_{2}$. By Theorem 2.1, the operator $A$ has a fixed point in $K \cap\left(\overline{\Omega_{2}} \backslash \Omega_{1}\right)$ such that $\rho_{1} \leq\|u\| \leq \rho_{2}$.

Theorem 3.2. Assume that $f_{0}=\infty$ and $f_{\infty}=0$. Then BVP (1.1) and (1.2) has at least one positive solution.

Proof. Since $f_{0}=\infty$, there exists $\rho_{1}>0$ such that $f(u) \geq \lambda u$, for $0<u \leq \rho_{1}$, where $\lambda>0$ is chosen so that

$$
\lambda \frac{\theta^{6}}{36} \cdot \frac{(1-\alpha+\beta)^{2}}{1-\alpha}(1-2 \theta)\left(\frac{1}{2}+\theta-\theta^{2}\right) \geq 1 .
$$


Thus, for $u \in K \cap \partial \Omega_{1}$ with

$$
\Omega_{1}=\left\{u \in C[0,1]:\|u\|<\rho_{1}\right\},
$$

we have from (3.2)

$$
\begin{aligned}
A u(t) & =\int_{0}^{1}\left(G(t, s)+\frac{1}{1-\alpha} \int_{0}^{1} a(\tau) G(\tau, s) d \tau\right) f(u(s)) d s \\
& \geq \int_{\theta}^{1-\theta}\left[\frac{\theta^{3}}{6} s(1-s)^{2}+\frac{1}{1-\alpha} \int_{\theta}^{1-\theta} \frac{\theta^{3}}{6} s(1-s)^{2} a(\tau) d \tau\right] \lambda u(s) d s \\
& \geq \lambda \frac{\theta^{6}}{36} \cdot \frac{(1-\alpha+\beta)^{2}}{1-\alpha}(1-2 \theta)\left(\frac{1}{2}+\theta-\theta^{2}\right)\|u\| \\
& \geq\|u\| .
\end{aligned}
$$

Then, $A u(t) \geq\|u\|$ for $t \in[\theta, 1-\theta]$, which implies that

$$
\|A u\| \geq\|u\|, \quad u \in K \cap \partial \Omega_{1} .
$$

Next we construct the set $\Omega_{2}$. We discuss two possible cases:

Case 1. If $f$ is bounded. Then, there exists $L>0$ such that $f(u) \leq L$. Set $\Omega_{2}=\left\{u \in C[0,1]:\|u\|<\rho_{2}\right\}$, where $\rho_{2}=\max \left\{2 \rho_{1}, \frac{L}{6(1-\alpha)}\right\}$.

If $u \in K \cap \partial \Omega_{2}$, similar to the estimates of (3.1), we obtain

$$
\begin{aligned}
A u(t) & \leq \frac{1}{6} \cdot \frac{L}{1-\alpha} \int_{0}^{1} s(1-s)^{2} d s \\
& \leq \frac{1}{6} \cdot \frac{L}{1-\alpha} \leq \rho_{2}=\|u\|,
\end{aligned}
$$

and consequently, $\|A u\| \leq\|u\|, \quad u \in K \cap \partial \Omega_{2}$.

Case 2. Suppose that $f$ is unbounded, since $f_{\infty}=0$, there exists $\widehat{\rho}_{2}>0$ $\left(\widehat{\rho}_{2}>\rho_{1}\right)$ such that $f(u) \leq \eta u$ for $u>\widehat{\rho}_{2}$, where $\eta>0$ satisfies

$$
\frac{\eta}{6(1-\alpha)} \leq 1
$$

On the other hand, from condition (H1), there is $\sigma>0$ such that $f(u) \leq \eta \sigma$, with $0 \leq u \leq \widehat{\rho}_{2}$.

Now, set $\Omega_{2}=\left\{u \in C[0,1]:\|u\|<\rho_{2}\right\}$, where $\rho_{2}=\max \left\{\sigma, \widehat{\rho}_{2}\right\}$.

If $u \in K \cap \partial \Omega_{2}$, then we have $f(u) \leq \eta \rho_{2}$. Similar to (3.1), we obtain

$$
\begin{aligned}
A u(t) & \leq \frac{1}{6} \cdot \frac{\eta \rho_{2}}{1-\alpha} \int_{0}^{1} s(1-s)^{2} d s \\
& \leq \frac{1}{6} \cdot \frac{\eta \rho_{2}}{1-\alpha} \leq \rho_{2}=\|u\|,
\end{aligned}
$$

so, $\|A u\| \leq\|u\|$, for $u \in K \cap \partial \Omega_{2}$. Therefore by Theorem 2.1, $A$ has at least one fixed point, which is a positive solution of (1.1) and (1.2). 


\section{Examples}

Example 4.1. Consider the boundary value problem

$$
\begin{gathered}
u^{\prime \prime \prime \prime}(t)+u^{2}\left(e^{-u}+1\right)=0, \quad 0<t<1, \\
u^{\prime}(0)=u^{\prime}(1)=u^{\prime \prime}(0)=0, u(0)=\int_{0}^{1} s^{2} u(s) d s,
\end{gathered}
$$

where $f(u)=u^{2}\left(e^{-u}+1\right) \in C([0, \infty),[0, \infty))$ and $a(t)=t^{2} \geq 0, \int_{0}^{1} a(s) d s=$ $\int_{0}^{1} s^{2} d s=\frac{1}{3}$.

We have

$$
\begin{aligned}
\lim _{u \rightarrow 0+} \frac{f(u)}{u} & =\lim _{u \rightarrow 0+} \frac{u^{2}\left(e^{-u}+1\right)}{u}=0, \\
\lim _{u \rightarrow+\infty} \frac{f(u)}{u} & =\lim _{u \rightarrow+\infty} \frac{u^{2}\left(e^{-u}+1\right)}{u}=+\infty .
\end{aligned}
$$

From Theorem 3.1, the problem (4.1) and (4.2) has at least one positive solution.

Example 4.2. Consider the boundary value problem

$$
\begin{gathered}
u^{\prime \prime \prime \prime}(t)+\sqrt{1+u}+\sin u=0, \quad 0<t<1, \\
u^{\prime}(0)=u^{\prime}(1)=u^{\prime \prime}(0)=0, u(0)=\int_{0}^{1} s u(s) d s,
\end{gathered}
$$

where $f(u)=\sqrt{1+u}+\sin u \in C([0, \infty),[0, \infty))$ and $a(t)=t \geq 0, \int_{0}^{1} a(s) d s=$ $\int_{0}^{1} s d s=\frac{1}{2}$.

We have

$$
\begin{aligned}
\lim _{u \rightarrow 0+} \frac{f(u)}{u} & =\lim _{u \rightarrow 0+} \frac{\sqrt{1+u}+\sin u}{u}=+\infty, \\
\lim _{u \rightarrow+\infty} \frac{f(u)}{u} & =\lim _{u \rightarrow+\infty} \frac{\sqrt{1+u}+\sin u}{u}=0 .
\end{aligned}
$$

Therefore, by Theorem 3.2, the problem (4.3) and (4.4) has at least one positive solution.

\section{Acknowledgement}

The authors would like to thank the anonymous referee for his valuable suggestions which lead to improve the manuscript. 


\section{References}

[1] E. Alves, T. F. Ma, and M. L. Pelicer, Monotone positive solutions for a fourth order equation with nonlinear boundary conditions, Nonlinear Anal., 71, (2009), 3834-3841.

[2] D. R. Anderson and R. I. Avery, A fourth-order four-point right focal boundary value problem, Rocky Mountain J. Math., 36, (2006), 367-380.

[3] J. R. Graef, C. Qian, and B. Yang, A three point boundary value problem for nonlinear fourth order differential equations, J. Math. Anal. Appl., 287, (2003), $217-233$.

[4] J. R. Graef and B. Yang, Existence and nonexistence of positive solutions of fourth order nonlinear boundary value problems, Appl. Anal., 74, (2000), 201-214.

[5] J. R. Graef, J. Henderson, and B. Yang, Positive solutions to a fourth-order three point boundary value problem, Discrete Contin. Dyn. Syst., Supplement, (2009), 269-275.

[6] X. Han, H. Gao, and J. Xu, Existence of positive solutions for nonlocal fourthorder boundary value problem with variable parameter, Fixed Point Theory Appl., 2011, (2011), Art. ID 604046, 11 pages.

[7] J. Henderson and D. Ma, Uniqueness of solutions for fourth-order nonlocal boundary value problems, Bound. Value Probl., 2006, (2006), Art. ID 23875, 12 pages.

[8] N. Kosmatov, Countably many solutions of a fourth-order boundary value problem, Electron. J. Qual. Theory Differ. Equ., 12, (2004), 1-15.

[9] M. A. Krasnoselskii, Positive Solutions of Operator Equations, P. Noordhoff, Groningen, The Netherlands, 1964.

[10] X. Liu and D. Ma, The existence of positive solution for a third-order two-point boundary value problem with integral boundary conditions, Scientific Journal of Mathematics Research, 4 (1), (2014), 1-7.

[11] R. Ma, Multiple positive solutions for a semipositone fourth-order boundary value problem, Hiroshima Math. J., 33, (2003), 217-227.

[12] R. Ma and W. Haiyan, On the existence of positive solutions of fourth-order ordinary differential equations, Appl. Anal., 59, (1995), 225-231.

[13] W. Shen, Positive solutions for fourth-order second-point nonhomogeneous singular boundary value problems, Adv. Fixed Point Theory., 5(1), (2015), 88-100.

[14] Y. Sun and C. Zhu, Existence of positive solutions for singular fourth-order threepoint boundary value problems, Adv. Difference Equ., 51, (2013), 13 pages.

[15] J. R. Webb, G. Infante, and D. Franco, Positive solutions of nonlinear fourthorder boundary value problems with local and nonlocal boundary conditions, Proc. Roy. Soc. Edinburgh Sect. A., 138, (2008), 427-446.

[16] B. Yang, Positive solutions for a fourth-order boundary value problem, Electron. J. Qual. Theory Differ. Equ., 3, (2005), 1-17. 
[17] Q. Yao, Local existence of multiple positive solutions to a singular cantilever beam equation, J. Math. Anal. Appl., 363, (2010), 138-154.

[18] S. Yong-Ping, Existence and multiplicity of positive solutions for an elastic beam equation, Appl. Math. J. Chinese. Univ., 3(26), (2011), 253-264.

[19] Q. Zhang, S. Chen, and J. Lü, Upper and lower solution method for fourth-order four-point boundary value problems, J. Comput. Appl. Math., 196, (2006), 387-393.

Slimane Benaicha

Laboratory of Fundamental and Applied Mathematics of Oran (LMFAO)

Department of Mathematics

University of Oran 1 Ahmed Benbella

31000 Oran

Algeria

E-mail: slimanebenaicha@yahoo.fr

Faouzi Haddouchi

Department of Physics

University of Sciences and Technology of Oran-MB

El Mnaouar, BP 1505

31000 Oran

Algeria

Laboratory of Fundamental and Applied Mathematics of Oran (LMFAO)

Department of Mathematics

University of Oran 1 Ahmed Benbella

31000 Oran

Algeria

E-mail: fhaddouchi@gmail.com

Received: 7.02.2016

Accepted: 16.05.2016 